\title{
A Hierarchical Structure for an Organic Chemistry Course
}

\author{
Jef Struyf \\ A retired instructor of the Health and Technology Department, Katholieke Hogeschool Leuven (KHLeuven), Belgium \\ *Corresponding author: jef.struyf@khleuven.be, jef.struyf@ucll.be
}

Received March 05, 2015; Revised March 29, 2015; Accepted April 29, 2015

\begin{abstract}
A teaching approach is proposed inspired by the hierarchical structure of the neon electron configuration. The course material is organized into analogous hierarchical 1s (molecular structure and physical properties), 2s (oxidation level based reaction schemes and acid-base) and 2p (three pairs of reaction mechanisms) course modules. The connection between acid-base and oxidation-reduction in organic chemistry is made clearer. General options, instructor's knowledge structure, building on prior knowledge, educational success, the value of the approach and the support from the educational research are discussed.
\end{abstract}

Keywords: organic chemistry, second-year undergraduate, course structure, analogies, acid-base, functional groups, neon, electron configuration, oxidation-reduction, oxidation level, reactions, mechanisms of reactions, mnemonics, physical properties

Cite This Article: Jef Struyf, “A Hierarchical Structure for an Organic Chemistry Course.” World Journal of Chemical Education, vol. 3, no. 2 (2015): 51-58. doi: 10.12691/wjce-3-2-5.

\section{Introduction}

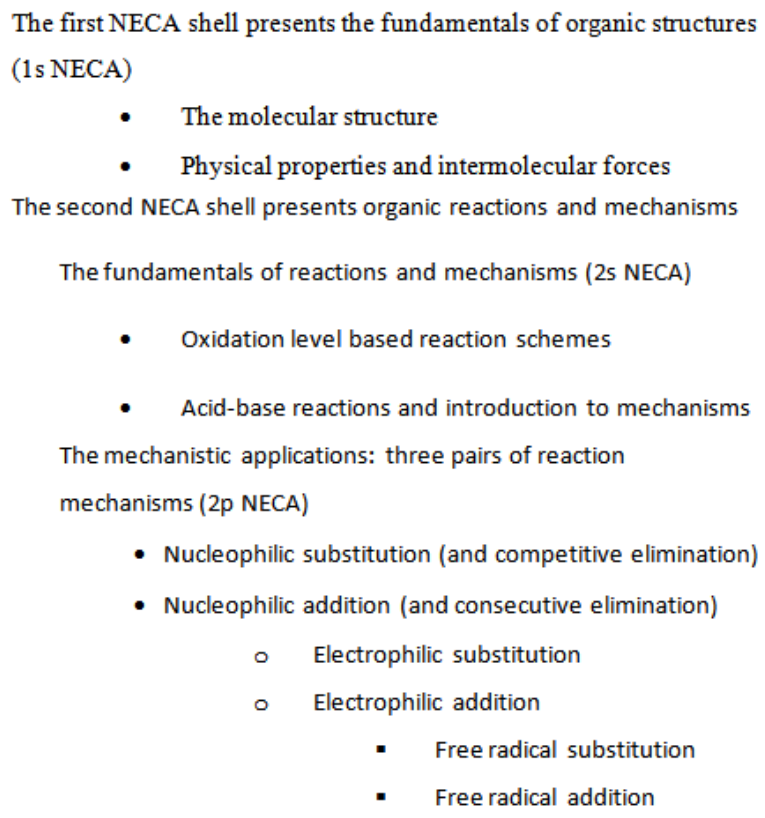

Figure 1. The Neon Electron Configuration Analogy (NECA) [12]

Reproduced with permission The Chemical Educator [http://chemeducator.org.

A major concern of textbook authors is the hierarchical structure of the course material. In most organic chemistry textbooks, the overall hierarchy is set by the course module sequence according to functional groups or reaction mechanisms. This article proposes a variant of the reaction mechanism approach $[1,2,3,4,5]$ that is potentially useful to instructors. It employs the familiar electron configuration of neon as a scaffold around which students' growing knowledge of organic chemistry can be organized. This approach presents an intriguing course structure that follows a Neon Electron Configuration Analogy (NECA). The analogy is already described in the Supplemental Material 2 of [12]. Reproduced with permission The Chemical Educator [http://chemeducator.org. The analogy is restricted to the main structure of the course. An outline of the course is shown in Figure 1. In NECA, organic chemistry topics are hierarchically organized in paired course modules. The NECA approach presents a simple and logical information hierarchy. Together with the 2s oxidation level based reaction schemes, NECA offers the students a relatively easy course overview, that results in an efficient scaffolding of their knowledge space [6]. The neon electron configuration is so well-known and the corresponding course material division so fundamental that it is strongly fixed in the mind of the students.

\section{The Neon Electron Configuration Structure of an Organic Chemistry Course}

\subsection{The 1s Shell of NECA: Molecular Structure and Physical Properties}

The 1s shell describes and discusses the molecular structure of organic compounds and their physical properties. For a given molecular structure, the corresponding experimental characteristics are their physical properties. Therefore, molecular structure and 
physical properties are directly linked (paired) and taught consecutively. The following reasoning provides further support for the content of the 1 s course shell. In the organic laboratory, we need compounds before we can proceed to explore reactions. Structure and physical properties are intrinsic features of a compound and researchers/students benefit from knowing them before proceeding to experiments involving reactions.

\subsubsection{Molecular Structure}

The molecular structure course module includes all the fundamental concepts of molecular structure. It describes electron distributions, names compounds and discusses their appearance in conformations, isomers, stereochemistry and 3D modeling. This module includes molecular architecture, which describes how molecules are designed by the bonding variability of the carbon atom and the octet rule. It relates functional groups to the periodic table.

\subsubsection{Functional Group Overview}

It is important for beginning students to have access to a well-structured functional group overview such as in [7]. It is not evident that beginning students can gain an overview of this knowledge from the in-depth studies in most textbooks. In [7], the connection to the periodic table and respectively to $\mathrm{CH}_{4}, \mathrm{NH}_{3}, \mathrm{H}_{2} \mathrm{O}$ and $\mathrm{HX}$ provides an appropriate functional group framework in which all functional groups covered by the course including their ionic derivatives are presented in Lewis and condensed formulas. This framework consists of six steps. Step 1: Single bonded functional groups. Step 2: Functional groups with multiple bonds to carbon including $\mathrm{R}^{1} \mathrm{COZ}$. Step 3: The $\mathrm{Z}$ variations of $\mathrm{R}^{1} \mathrm{COZ}$. Step 4: (Hemi)acetals, (hemi)ketals, imides and anhydrides. Step 5: Organic derivatives of $\mathrm{HNO}_{2}, \mathrm{H}_{3} \mathrm{PO}_{4}$ and $\mathrm{H}_{2} \mathrm{SO}_{3}$. Step 6: (Supplemental Information of [7]): The thio derivatives and the organometallic compounds. Introductory nomenclature can be easily connected to the functional group framework. An example table, Table 1 corresponding to step 1 , is included with the permission of the Journal of Chemical Education.

Table 1. Single bonded functional group classes and their ionic forms in relation to the periodic table [7]

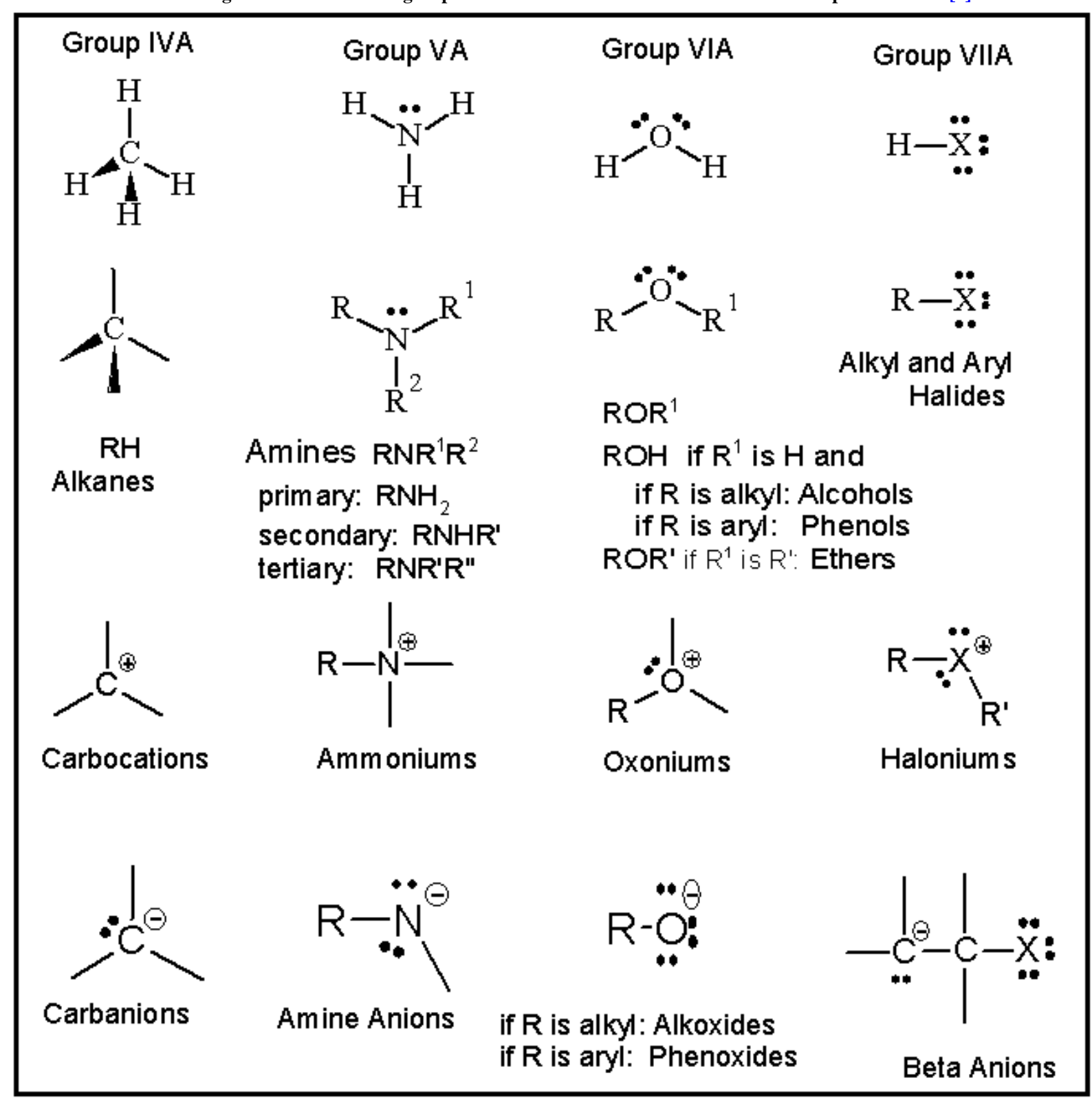

Reproduced with permission of the Journal of Chemical Education. 


\subsubsection{Training of the Functional Group Overview}

To support efficient communication, students can relate class names to structures and structures to class names right from the beginning of the course. In this part of the course, the playing cards analogy is crucial. If you want to learn organic chemistry you have to learn about the different cards, the functional groups, of the game. Training of the functional group framework early in the course is painful but inevitable. Underestimating this fundamental course part can be disastrous for many students.

Table 2. Related Properties for Selected Compounds of the Compared Homologous Series in Figure 2 [8]and [17]

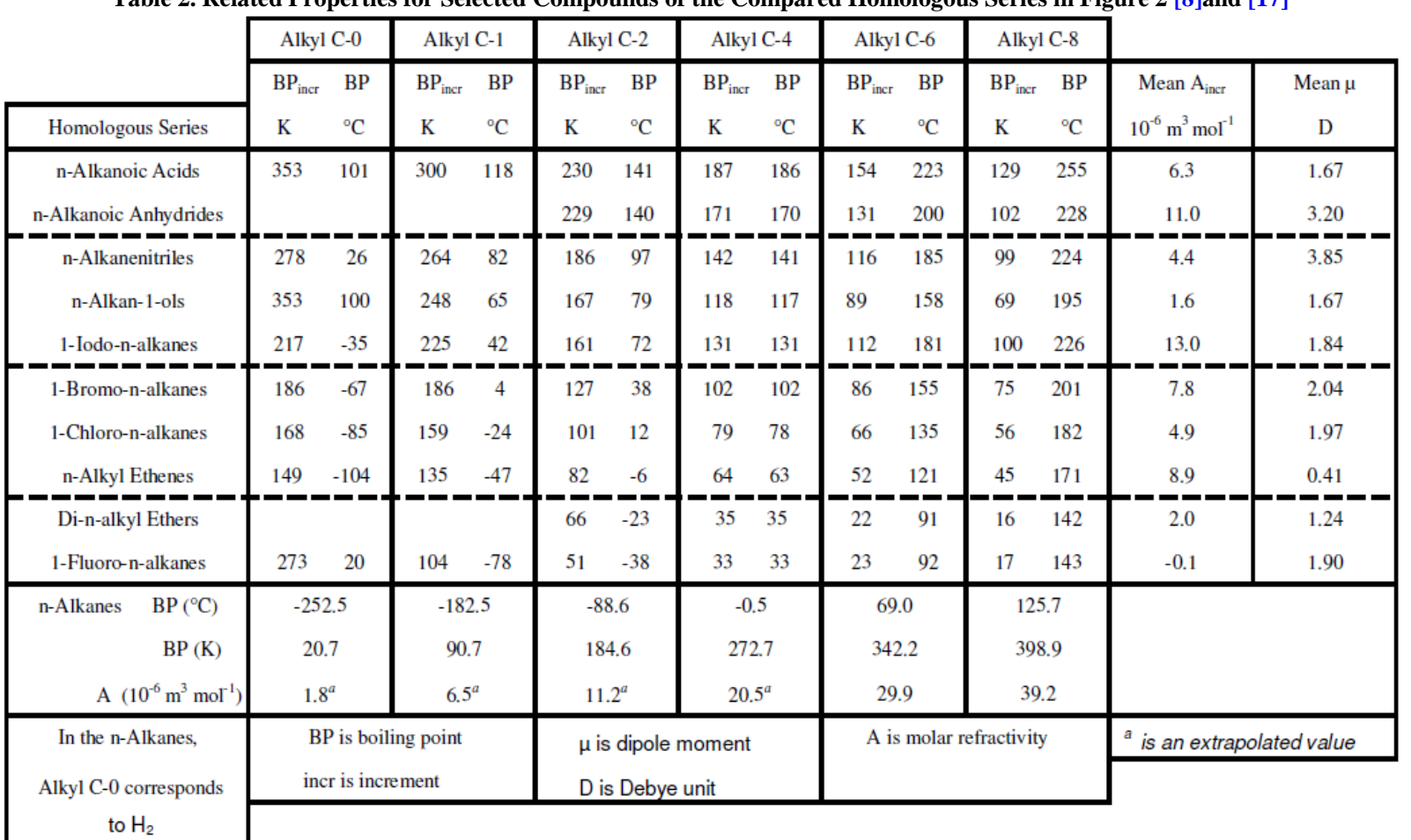

Reproduced with permission of the Journal of Chemical Education.

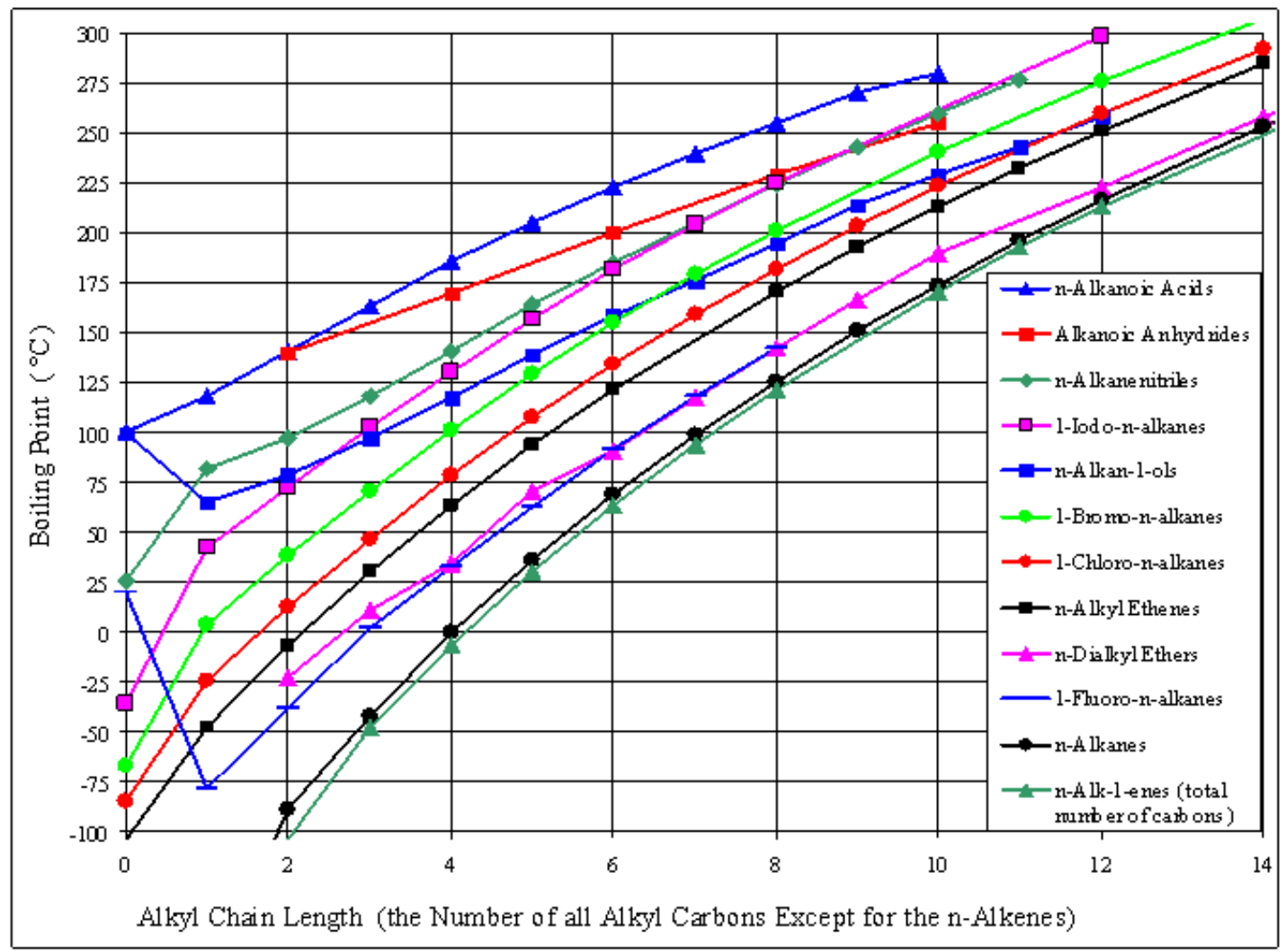

Figure 2. Overview of Some Boiling Point Curves [9] and [17] 


\subsubsection{Physical Properties}

The physical properties course module not only covers melting and boiling points but also the spectrometric structure determination tools. Especially the boiling points are very interesting for a structure-property comparison and make organic structures more real by relating the abstract formulas to a concrete measurable property (the boiling point). Reference [8] proposes an analytical approach for relating boiling points of monofunctional compounds to intermolecular forces. The boiling point of a compound is the sum of a contribution due to its Rgroup(s) and a contribution due to the functional group. The contribution of the functional group decreases with the alkyl chain length which indicates masking of the functional group by the alkyl group(s). The contribution of the functional group is calculated from boiling point differences to the corresponding alkanes (the boiling point increment) and is computed from the dipole moment and the molar refraction of the functional group (the molar refraction increment). Molar refraction represents the London dispersion forces and the dipole moment the dipole-dipole interactions. The boiling point curves in [9] are an important material for presenting this subject. Figure 2 shows an example graph. From such graphs and in more detail from the corresponding data Table 2 students can gain insight in the relative strength of the intermolecular forces for compounds in homologous series of the various functional groups of the course. At selected alkyl chain length(s), we can ask the students to relate the boiling point data to molecular structure and corresponding intermolecular forces. In view of assessments (ii) and (iii) of [10] concerning this structure-property connection, example connections between structures, data and forces are advisable also as a recapitulation at the end of the course. Table 2 and Figure 2 are reproduced with permission of the journal of chemical education.

\subsection{The 2s Subshell of NECA: Oxidation Level Based Reaction Schemes and Acid-Base Reactions}

The second shell electrons of carbon are involved in organic reactions, and similarly the second NECA information shell targets organic reactions. The 2s electrons, like the $1 \mathrm{~s}$ electrons, have a spherical orbital. In the NECA hierarchy, the spherical feature corresponds to fundamentals. Accordingly, the 2s NECA subshell provides the fundamentals of reactions and their mechanisms. This subshell discusses reactions that are necessary for an optimal study of reaction mechanisms (at the 2p NECA subshell). These reactions are oxidationreduction and acid-base. General chemistry courses thoroughly discuss oxidation-reduction and acid-base. The 2s NECA subshell extends them to organic compounds. They are excellent starting points for other organic reactions and their mechanisms. The choice of covering oxidation-reduction and acid-base reactions together at the 2s NECA subshell is further supported by the fact that both reactions can be split into half-reactions. This common property pairs them together and puts oxidationreduction and acid-base reactions in line with the NECA structure. The 2 s electrons are similar to the 1 s electrons, but reside on a higher energy level. In a similar way, oxidation-reduction and acid-base reactions (2s subshell) build upon oxidation state and formal charges, which have already been encountered at the molecular structure 1s NECA shell. Both 1s concepts are integrated in the corresponding 2s reactions.

\subsubsection{Oxidation Level Based Reaction Schemes}

When students entered the organic chemistry reaction field, the clue position of the oxidation-reduction reactions may become less clear to them. Student can have the impression that these reactions are not more than unimportant footnotes. Many, perhaps most textbooks did not use the oxidation level (OL) concept. By this, it is nearly impossible to make clear that all other reactions occur at well-defined OLs. Organic chemistry reactions are in between the OL for hydrocarbons and the $\mathrm{CO}_{2} \mathrm{OL}$.

NECA proposes the use of oxidation-reduction at this intermediate NECA subshell for the following reason. In traditional textbooks, the sequence of the functional group course modules nearly follows the OLs of the functional group (attached) carbon(s). It is a good educational practice to explicitly communicate this knowledge to our audience optimally in the form of basic Reaction Scheme 1 (Figure 3). The scheme shows strategically chosen functional group conversion reactions for a first, elementary overview of the reaction landscape in a wellstructured way. Two properties of the functional groups are appropriate for constructing the basic Reaction Scheme 1: functional groups can be related to the periodic table [7] and they can be classified by OL [4,11]. Oxidation-reduction reactions form the vertical dimension of the scheme. The scheme is the core of the hierarchy [12] because the mental process required from the students to relate other reactions and mechanisms to this central scheme results in a coherent knowledge space. The scheme is intrinsically but never explicitly present in textbooks. Implicitly, the various OLs are the backbone of every organic chemistry course and this 2s basic reaction scheme makes it explicit. Some reactions of the scheme have side reactions such as the products of the carbocation rearrangements. Side reactions confront students with the shortcomings of the scheme. A more comprehensive approach including reaction mechanisms will be necessary. More details and instructional aids are available in the Supplemental Information. The 2s NECA subshell allows for additional schemes (Supplemental Information) based on OLs resulting in a complete overview of most reactions covered by the course. Each additional scheme shows at least one functional group (in bold font) that connects to the basic Reaction Scheme 1. The schemes guide the student in the possibly overwhelming field of reactions. The additional schemes are already advisable at the 2s NECA subshell because the students are used to see most functional groups of the course in each 1s NECA topic and acid-base. They help the students to develop their knowledge space on reactions. The reaction overview focuses on the functional group conversions and not yet on mass balance and stoichiometric correctness. Oxidation-reduction has already been proposed for a logical characterization of organic chemistry structures as part of a three-dimensional reaction overview by type [13].

\subsubsection{Acid-Base Reactions}

The choice of acid-base as a starting point for organic reactions is generally accepted due to the fact that acids or 
bases catalyze many reactions. This is also the best occasion to introduce the curved arrows for writing reaction mechanisms. The standard two-arrow pattern allows the students to focus on what really happens when using arrows, not disturbed by mechanistic complications such as in nucleophilic substitution at saturated carbon.

The reaction knowledge space is at best three dimensional. The acid-base properties of the functional groups extend the basic Reaction scheme 1 to a basic reaction knowledge space. Figure 4 shows a three dimensional graph corresponding to Reaction scheme 1 with the pKa values [14,15] of the functional groups in the z-dimension. In addition, alkyl and aryl derivatives of some functional groups and the alkyloxoniums are included. Acid-base reactions in the z-dimension of the scheme are in accordance to their applications. They are not intended to create a new functional group, but change a compound's reactivity or solubility.

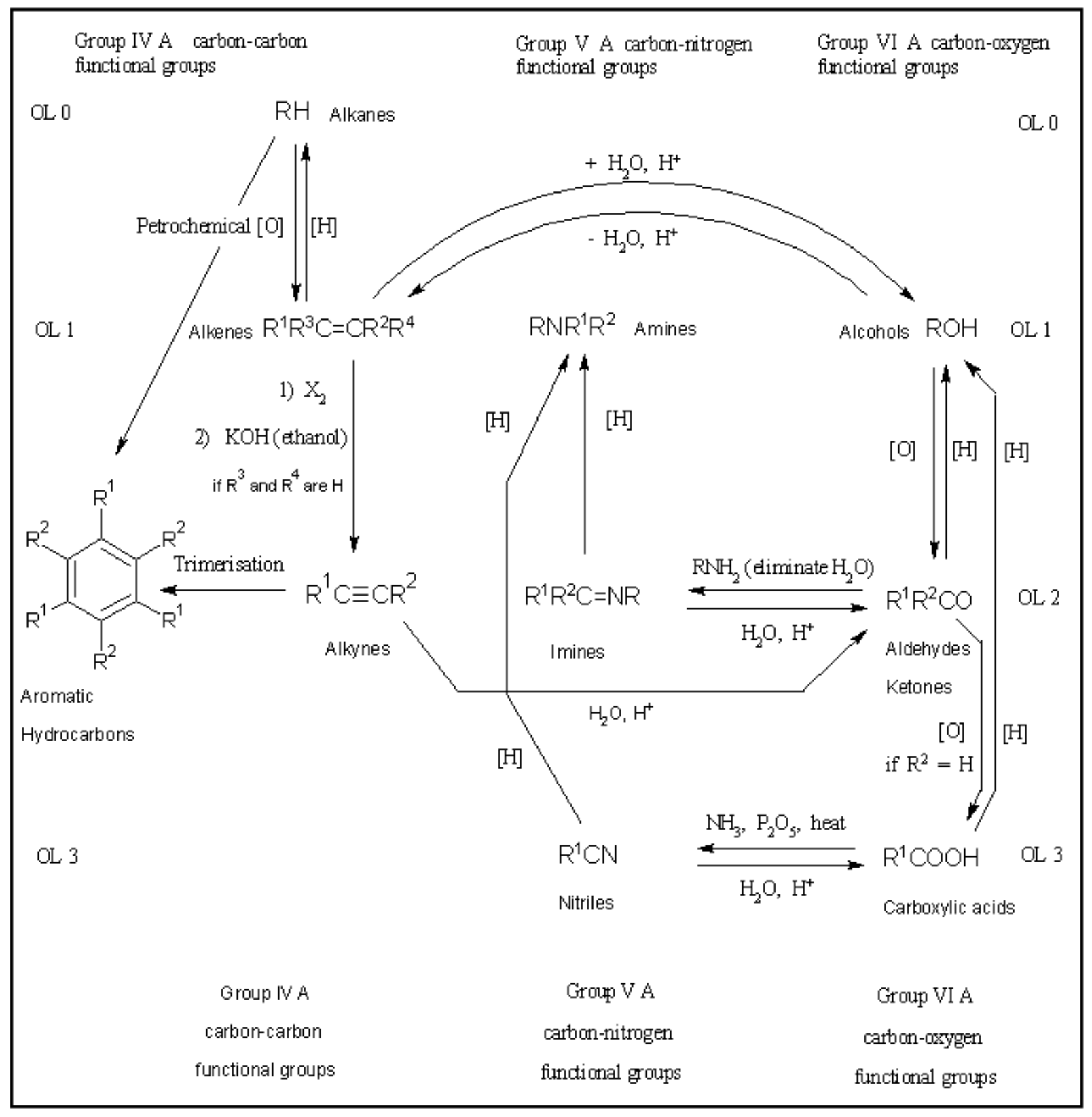

Figure 3. Reaction Scheme 1. The 2s NECA basic organic reaction scheme, which is ordered by periodic table connections and oxidation levels (OLs). Groups IVA, VA and VIA indicate the position of the most specific (hetero) atom of a functional group in the periodic table. $R^{1}, R^{2}, R^{3}$ and $R^{4}$ are used to enumerate the R groups or to denote hydrogen. The numbers 1) and 2) next to a reagent indicate consecutive reactions [12].

Reproduced with permission The Chemical Educator, http://chemeducator.org.

\subsubsection{Total Approach in 1s and 2s NECA}

Both s NECA (sub)shells form a foundation on which further study of organic reactions, mechanisms and the planning of synthetic pathways can be based. Studying the s (sub)shells is like playing cards. One must first learn what the cards look like by learning structural formulas [7]. After that one can start playing the simplest games. For example: nomenclature, physical properties, isomers, stereochemistry, acid-base ..., while learning the basic rules. The learning process integrates as many functional groups as possible in each 1 s and 2s topic, which I call a total approach. My articles [7,8,9] show how a total approach can be developed for the corresponding course topics. In most textbooks based on functional group course modules; nomenclature, physical properties and structures are scattered over the course modules. 


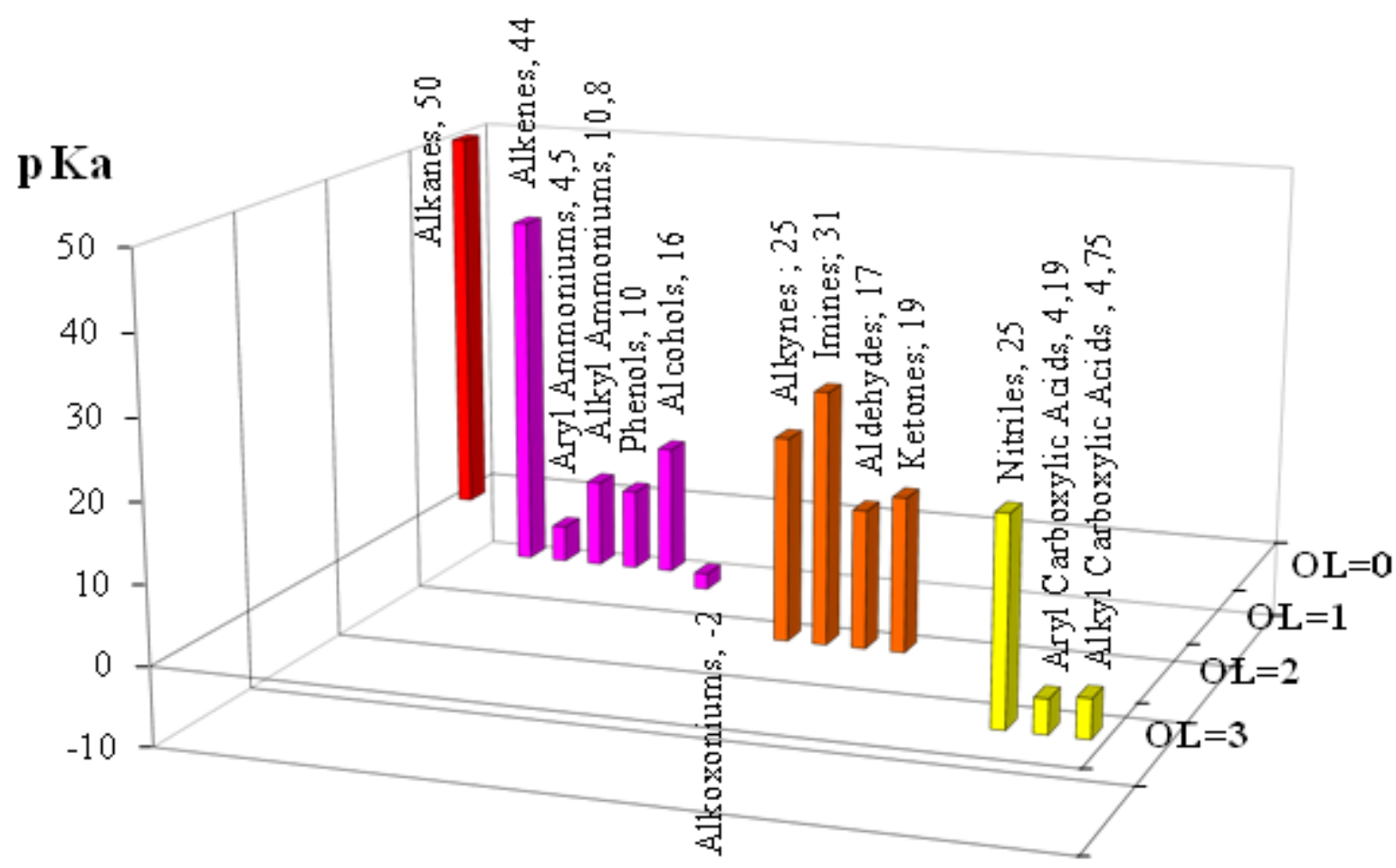

Figure 4. A 3-D graph corresponding to Reaction scheme 1 showing the pKa values of the functional groups [14,15] and [17]

\subsection{The 2p NECA Subshell}

\subsubsection{Three Paired Reaction Mechanisms}

The 2p subshell is dedicated to reaction mechanisms. There are three main mechanisms: nucleophilic, electrophilic and free radical. Each mechanism has an addition and a substitution variant forming one mechanism pair. This choice is obvious for the electrophilic and free radical mechanisms, but less obvious for nucleophilic mechanisms and eliminations. Elimination reactions mostly are in competition with nucleophilic substitution reactions on saturated carbon and in most cases they immediately follow nucleophilic addition (nucleophilic addition and consecutive elimination). Elimination is a feature of nucleophilic reactions that in both cases can be suppressed by substrate structure. For example, the absence of $\beta$-hydrogen atoms in alkyl halides, or the absence of a suitable leaving group in aldehydes and ketones. Consequently, these mechanisms are also considered one pair (Figure 1). Rearrangement reactions are nucleophilic, free radical or electrophilic in mechanism [14]. Although rearrangement reactions have very specific and interesting products, they show no new ideas in the mechanistic field.

\subsubsection{NECA Hybridization}

In carbon compounds, the $2 \mathrm{~s}$ and $2 \mathrm{p}$ electron subshells are hybridized in chemical bond formation. In analogy, an acid or base catalyst reacts ("hybridizes") with a reactant at the 2p NECA subshell assisting in product formation. How oxidation-reduction reactions interact ("hybridize") with 2p mechanisms becomes clear in the use of Reaction scheme 1 and its connecting schemes (supplemental information). The OLs and the functional groups in reaction schemes form the framework to which the reaction mechanisms are linked to construct the knowledge space. The link is of such a nature that the mechanisms are implicitly part of the schemes (hybridized), because reactions are all based on some mechanism.

\section{Discussion}

\subsection{General Options of NECA}

The basic concern of the NECA approach is to improve the insight of the students in the course material. The author teaches NECA, which is originally intended for the first year of a two-year course, taking into account a few main principles. He teaches the fundamentals first and informs the students about his knowledge structure and discusses the mechanisms later on. He challenges the students by structure-property problems and later on by reaction-mechanism connections. The troublesome nucleophilic substitution at saturated carbon and competitive elimination is best saved for the second year of the course.

\subsection{Instructors' Knowledge Structure}

The difference between this NECA course and similar approaches is in making the instructors' knowledge structure more explicit. The development of the students' knowledge on the overwhelming variety in molecular formulas and reactions covered by the course are two parts of the instructors' knowledge structure, which students need to develop for themselves during their own struggle with the course material. The reaction overview is the $2 \mathrm{~s}$ 
NECA equivalent of the functional group overview in $1 \mathrm{~s}$ NECA. The functional group overview demonstrates their structural relations; the reaction overview additionally shows the reaction conversions of functional groups. In the reaction overview by schemes, oxidation levels are the scaffold and acid-base forms the third dimension. All this information from the instructors' knowledge structure is not explicitly included in textbooks, which makes it difficult to pass this knowledge on to the students. A NECA course explicitly informs the students about this knowledge structure and connects thereby on prior knowledge, especially in the molecular structure and the 2s NECA subshell course modules.

\subsection{Building on Prior Knowledge}

Functional groups are like cards or chess pieces. We learn about them by playing and start each game at a level that connects to prior knowledge. In the formula overview as well as in the reaction overview, NECA connects to the well-known periodic table. Students have to expand their knowledge structure by building on prior knowledge. They increase their knowledge by adding fundamental concepts. They master the fundamentals by structureproperty connections (games). It is not wise to distract the students at this level with the overwhelming reactionmechanism field. When entering the latter field, first gradually show the reaction landscape (overview) in which they have to operate (2s NECA). Like the electrons of carbon, nitrogen and oxygen atoms, functional groups are only involved in reactions at the second (NECA) shell.

\subsection{Educational Success}

The educational success of instructors also depends on a strong believe in their approach and on whether they have good stories to motivate students. NECA is excellent in that respect. When reaching the $2 p$ mechanisms, the motivating power of NECA decreases. Its orienting possibilities are now supported in addition by the reaction overview developed at $2 \mathrm{~s}$ NECA. Furthermore, the instructor may become creative with stories to sustain students' attention. Although all of organic chemistry is basic to biochemistry, the carbonyl chemistry is outstanding in that respect. Carbonyl chemistry is the highway to biochemistry. In this context, one can use the mnemonic device for carbonyl reactions that the author has developed [[12], pp. 7-8]. The device resembles the human body. To explain the orientation rules of electrophilic substitution, the analogy of a lover's date can be amusing and at the same time focus the students' attention: "Love (orientating group), tell me (electrophilic reagent) when (with the appropriate catalyst) and where (ortho-para or meta position)". A love story appeals to the sensations of the heart, like the heart of an introductory organic chemistry course. (Love date is Reproduced with permission The Chemical Educator, http://chemeducator.org.).

\subsection{Value of NECA}

The value of NECA is that all organic chemistry contents can be sorted in the NECA hierarchy. NECA proposes a matching hierarchical scaffold for students learning that connects to prior knowledge. The hierarchy separates the general fundamentals (1s NECA) from the reaction mechanism fundamentals (2s NECA). NECA makes the connections between oxidation-reduction and acid-base as well as between nucleophilic and elimination mechanisms much clearer. It is motivating for students to know that organic chemistry can be organized as NECA; it stimulates their eagerness to learn. The tables and graphs in the author's publications $[7,8,9]$ and the reaction schemes in [12], in this article and its supplemental information including instruction aids, may stimulate students to develop specific topic and course overviews by independent study.

The question remains if the course will result in better student results. There are pronounced positive long term results published [[12], p.9] by comparing the NECA students in the second year of a two-year course with nine control groups. The NECA course was in the first year. It would be interesting if these results could be independently confirmed by an appropriately designed chemical education research study.

\subsection{Support from the Chemical Education Research Literature}

The chemical education research literature supports the efficacy of the curriculum and the need to scaffold students' learning in a hierarchical way. Cooper [16] records that "Learning materials that are designed with an understanding that meaningful learning builds upon and connects to students' prior knowledge and that difficult concepts are best developed in a well-designed scaffold framework or learning progression are far more likely to help students develop a robust understanding of chemical concepts". Taagepera and Noori [6] point out that "students' cognitive organization of the knowledge was surprisingly weak" and stress the importance of hierarchy in the introduction of organic chemistry concepts. "Instructors need to be more aware of their own knowledge structure and make it clearer to the students". This is precisely what NECA tries to accomplish.

Concerning the 1s NECA shell, Cooper et al. [9] found that: "(i) many students were unable to construct representations of simple molecular structures; (ii) a majority of students fail to make the important connection between these representations and macroscopic properties of the material; and (iii) they were unable to decode the information contained in such representations". Therefore Cooper et al develop "The clue approach to structure and function: a learning progression ${ }^{10}$,. The author agrees with assessments (ii) and (iii) and is convinced that the clue approach helps to overcome these difficulties. Students need challenging topics; we should not avoid those difficulties. Assessment (i) is not a problem in the NECA practice.

\section{Conclusion}

A NECA hierarchical course structure is presented that may help students in their organic chemistry adventure. This approach proceeds more gradually from the simple to the more complex. The oxidation level structure and the connection between acid-base and oxidation-reduction in organic chemistry are made clearer Some parts of the instructor's knowledge structure become more explicit. NECA is in line with educational research results. 


\section{Supplemental Information}

The Supplemental Information discusses oxidation levels and the basic Reaction scheme 1 more in detail including the instructional aids to present it to the students and shows its connecting schemes for constructing a reaction overview of the course.

\section{Abbreviations}

$\mathrm{OL}$ Oxidation Level

NECA Neon Electron Configuration Analogy.

\section{References}

[1] Chaloner, P. Organic Chemistry, a Mechanistic Approach, Apple Academic Press: Oakville; 2014.

[2] Bruice, P.Y. Organic Chemistry, $7^{\text {th }}$ ed.; Prentice Hall: Upper Saddle River; 2013.

[3] Okuyama, T.; Maskill, H. Organic Chemistry, a Mechanistic Approach, Oxford University Press: Oxford; 2013.

[4] Clayden, J.; Greeves, N.; Warren, S. Organic Chemistry, $2^{\text {nd }}$ ed.; Oxford University Press: Oxford; 2012.

[5] Fox, M.A.; Whitesell, J.K. Organic Chemistry, $3^{\text {th }}$ ed.; Jones and Barlett Publishing Company: London; 2004.
[6] Taagepera, M.; Noori, S. Mapping Students' Thinking Patterns in Learning Organic Chemistry by the Use of Knowledge Space Theory. J. Chem. Educ., 2000, 77 (9), 1224-1229.

[7] Struyf, J. Relating Functional Groups to the Periodic Table. $J$. Chem. Educ., 2009, 86 (2), 190-193.

[8] Struyf, J. An Analytical Approach for Relating Boiling Points of Monofunctional Organic Compounds to Intermolecular Forces. $J$. Chem. Educ., 2011, 88 (7), 937-943.

[9] Struyf, J. Comparative Graphs for Boiling Point Curves of Representative Homologous Series. J. Chem. Educ., 2012, 89 (6), 819-820.

[10] Cooper, M.; Underwood, S.; Hilley, C.; and Klymkowsky, M. Development and Assessment of a Molecular Structure and Properties Learning Progression. J. Chem. Educ., 2012, 89 (11), 1351-1357.

[11] Charonnat, J. Chemistry 333 http://www.csun.edu/ hcchm007/333oloc.pdf (accessed June 2014).

[12] Struyf, J. Oxidation-Reduction as the Core of a Total Approach Introductory (Bio) Organic Chemistry Course Inspired by the Neon Electron Configuration. The Chem. Educator, 2007, 12 (1), $1-9$

[13] Hendrickson, J.B. Logical Characterization of Organic Chemistry. J. Chem. Educ. 1978, 55(4), 216-221.

[14] March, J. Advanced Organic Chemistry, $4^{\text {th }}$ ed.; John Wiley, New York: 1992; pp 250-252.

[15] Bordwell pKa Table, http//www.chem.wisc.edu/areas (accessed May 2014)

[16] Cooper, M. The New MCAT: An Incentive for Reform or a Lost Opportunity? J. Chem. Educ., 2013, 90 (7), 820-822.

[17] Lide, D.R. Handbook of Chemistry and Physics, 90th ed.; CRC Press: Boca Raton, FL, 2009-2010. 